\title{
Vision and visual plasticity in ageing mice
}

\author{
Konrad Lehmann*, Karl-Friedrich Schmidt ${ }^{1}$ and Siegrid Löwel ${ }^{1, *}$ \\ Institut für Allgemeine Zoologie und Tierphysiologie, Friedrich-Schiller-Universität Jena, Jena, Germany
}

\begin{abstract}
Purpose: Little is known about neuronal changes during ageing in the visual system of mice which are increasingly being used as animal models for human visual disorders.

Methods and results: Measuring the optomotor response to moving gratings, visual acuity of C57BL/6-mice was 0.39 cycles/degree (cyc/deg) until 12 months of age and declined to $0.27 \mathrm{cyc} / \mathrm{deg}$ (by 30\%) at 26 months. In the visual water task, a cortex-dependent task based on visual discrimination learning, visual acuity remained stable at 0.58 cyc/deg up to 21 months and then declined to 0.48 cyc/deg (by 19\%) at 27 months. Visual cortical activity recorded by optical imaging declined by $33 \%$ between seven and 23 months of age. After monocular deprivation and daily testing of the optomotor response, visual acuity of the open eye increased by $29 \%$ in 4 to 7 -month-old animals, while the increase was only $13 \%$ in 23 -month-old mice. Interestingly, interindividual variability generally increased with age, so that some 23 -month-old mice retained visual acuity and interocular plasticity like 4 or 7-month-old animals.

Conclusions: In summary, reduced visual function was accompanied by a reduction of both visual cortical responses and interocular plasticity indicating a central nervous system component in age-related vision loss in mice.
\end{abstract}

Keywords: Visual cortex, optomotry, visual water task, optical imaging, sensory learning

\section{Abbreviations}

$\begin{array}{ll}\text { ANOVA } & \text { analysis of variance } \\ \text { MD } & \text { monocular deprivation } \\ \text { ODI } & \text { poular dominance index } \\ \text { PD } & \text { visual acuity asymmetry index } \\ \text { VAAI } & \text { four/seven/twelve/18/23/26 } \\ 4 \mathrm{M} / 7 \mathrm{M} / 12 \mathrm{M} / & \text { months of age }\end{array}$

\footnotetext{
${ }^{1}$ Present Address: Systems Neuroscience, Bernstein Focus Neurotechnology (BFNT) and Johann-Friedrich-Blumenbach Institut für Zoologie und Anthropologie, Georg-August-Universität Göttingen, Göttingen, Germany.

${ }^{*}$ Corresponding author: Konrad Lehmann, PhD, Institut für Allgemeine Zoologie und Tierphysiologie, Friedrich-SchillerUniversität Jena, Erbertstr. 1, D-07743 Jena, Germany. Tel.: +49 3641949 106; Fax: +49 3641949 102; E-mail: Konrad.Lehmann@ uni-jena.de; and Siegrid Löwel, PhD, Systems Neuroscience, Bernstein Focus Neurotechnology (BFNT) and Johann-FriedrichBlumenbach Institut für Zoologie und Anthropologie, GeorgAugust-Universität Göttingen, Göttingen, Germany. Tel.: +49 551 39 20161; Fax: +49 55139 20162; E-mail: sloewel@gwdg.de.
}

\section{Introduction}

In old age, many people experience a decline in visual capabilities, including acuity and contrast sensitivity (Frisén and Frisén, 1981; Owsley et al., 1983; Elliott et al., 1995; Ferrer-Blasco et al., 2008). In ageing humans, the perceptual impairments go along with alterations in physiological measures: In the electroretinogram, the b-wave amplitude indicating interneuronal activity was reduced (Weleber, 1981; Birch and Anderson, 1992), as was the amplitude of visually evoked potentials (Tomoda et al., 1991; Porciatti et al., 1992). However, changes in ocular transmission or retinal processing cannot account for all observed impairments, and in consequence, an involvement of the visual neural pathways has been suggested (see Spear, 1993 for review). Indeed, a decreased signal-to noise ratio and reduced orientation and direction selectivity in the visual cortices of aged monkeys, cats and rats have been shown (Schmolesky et al., 2000; Hua et al., 2006; Wang et al., 2006). 
Much less is known about the visual abilities and visual system of ageing mice, although the mouse, as it allows for a large variety of genetical, pharmacological and surgical manipulations, is increasingly being used as the standard animal model for research on the developing and ageing visual system. In addition, most current research on the plasticity of the visual system is being conducted in this animal (Hensch, 2005). In the C57BL/6 strain, several studies have described reduced electroretinogram amplitudes during ageing, while retinal morphology apparently remained unchanged ( $\mathrm{Li}$ et al., 2001; Gresh et al., 2003; Williams and Jacobs, 2007). Visual acuity, as measured by a discrimination task, declined from 0.48 cycles/degree (cyc/deg) at an age of 6 months to $0.38 \mathrm{cyc} / \mathrm{deg}$ in two-year-old mice (Wong and Brown, 2007). The gain of the optokinetic response decreased already between four and nine months of age in male mice (van Alphen et al., 2009). The behavioural increment thresholds of photopic and scotopic vision, in contrast, have been shown to be completely independent of age (Williams and Jacobs, 2007).

Together, these studies have suggested that pigmented mice are a suitable model for investigating age-related changes in the visual system, but more data are needed on the development of visual abilities throughout life. Since a detailed understanding of the mechanisms underlying age-related vision loss may help to develop preventive or restorative treatments, we set out to use a combination of behavioural and imaging experiments to study both visual performance and visual cortical activity patterns in individual mice at many time points throughout life. To this end, we used two different behavioural tests in mice, optomotry, a virtual-reality optomotor system (Prusky et al., 2004), and the visual water task (VWT; (Prusky et al., 2000) to measure visual acuity and contrast sensitivity in mice from 4 to 26 months of age. Moreover, we addressed the question of a cortical involvement in age-related visual decline that has been raised in humans. Since it was recently shown that the visual acuity of the open eye increased after monocular deprivation (MD) and daily testing in an optomotor setup (Prusky et al., 2006), and that this increase is dependent on the cortex, we also tested this "interocular plasticity" (Prusky et al., 2006) in animals of a larger age range, in particular including twoyear-old animals. Finally, we directly analyzed visual cortical activity patterns in ageing mice by using optical imaging of intrinsic signals (Cang et al., 2005a; Lehmann and Löwel, 2008): we visualized cortical responses and retinotopic maps in mice of various ages, determined the magnitude of cortical activation upon visual stimulation and cortical plasticity after monocular deprivation.

\section{Materials and methods}

\subsection{Animals and rearing conditions}

C57BL/6 mice were raised in standard cages on a $12 \mathrm{~h}$ light/dark cycle, with food and water available $\mathrm{ad}$ libitum. In addition to our own breeding colony, aged animals were obtained from the animal facilities of the Leibniz Institute for Neurobiology (Magdeburg) and the university clinic in Jena. Before each experiment, the animals' eyes were inspected with an ophthalmoscope. Animals in which corneal damage was detected were excluded from the experiments.

For our cross-sectional study, age groups were defined as follows: Four months (4M) old, including mice from postnatal day (PD) 117 to 153 at the beginning of the experiments, 7M: PD 207-238, 12M: PD 347-368, 18M: PD 538-544, 23M: PD 592-757 (19 to 25 months), 26M: PD 776-824 (25-27 months). All animals in the $4 \mathrm{M}, 7 \mathrm{M}, 12 \mathrm{M}, 18 \mathrm{M}$ and $26 \mathrm{M}$ groups were males. Animals in the $23 \mathrm{M}$ group were of both sexes. In humans, sex has an influence on visual acuity, with women enjoying better vision than men (FerrerBlasco et al., 2009). In the present study, we exclusively used male mice in the younger age groups, but included some females in the $23 \mathrm{M}$ (six out of $32,19 \%$ ) group. Visual acuity and contrast sensitivity of our mice were - however - not influenced by sex $(p=0.71$, $t$-test).

Assessment of visual acuity and contrast sensitivity in the virtual-reality optomotor system was performed in all animals of all age groups. Interocular plasticity after monocular deprivation (MD) and visual cortical maps were studied in a subset of $4 \mathrm{M}, 7 \mathrm{M}$ and $23 \mathrm{M}$ animals. In 23M mice, we used both control mice (without MD) and mice with MD for optical imaging of intrinsic signals. All animals analysed with imaging were also tested by optomotry. Table 1 gives an overview of the number of animals used in the different procedures.

Additionally, visual acuity was followed longitudinally in a separate group of ten male mice from twelve 
Table 1

Number of mice analysed with the various techniques in the 6 different age groups (4M, 7M, 12M, 18M, 23M, 26M: 4, 7, 12, 18, 23 and 26 months of age on average)

\begin{tabular}{lcccccc}
\hline & $4 \mathrm{M}$ & $7 \mathrm{M}$ & $12 \mathrm{M}$ & $18 \mathrm{M}$ & $23 \mathrm{M}$ & $26 \mathrm{M}$ \\
\hline Age (days) & $117-153$ & $207-238$ & $347-368$ & $538-544$ & $592-757$ & $776-824$ \\
Optomotry $(n)$ & 10 & 31 & 10 & 6 & 32 & 15 \\
Interocular plasticity $(n)$ & 7 & 12 & & & 11 & \\
Optical imaging & & & & & 8 \\
$\quad$ Without MD $(n)$ & 4 & 8 & & & 8 \\
$\quad$ With MD $(n)$ & - & - & & & \\
\hline
\end{tabular}

Table 2

Visual acuity, standard errors and sample sizes of the mice investigated longitudinally in the visual water task and the virtual-reality optomotor system

\begin{tabular}{|c|c|c|c|c|c|c|}
\hline Age (months) & 12 & 15 & 18 & 21 & 24 & 27 \\
\hline$n$ & 10 & 10 & 10 & 8 & 5 & 4 \\
\hline VWT visual acuity (cy & $0.48 \pm 0.01$ & $0.56 \pm 0.02$ & - & $0.58 \pm 0.02$ & $0.52 \pm 0.02$ & $0.48 \pm 0.03$ \\
\hline Optomotry visual acuity (cyc/deg) & $0.38 \pm 0.001$ & $0.38 \pm 0.001$ & $0.36 \pm 0.002$ & $0.35 \pm 0.003$ & $0.33 \pm 0.008$ & $0.33 \pm 0.01$ \\
\hline
\end{tabular}

to 27 months of age. These animals were tested by both optomotry and in the visual water task. Measurements were performed every three months. Table 2 gives an overview of these animals and the timepoints of measurement.

All experimental procedures have been performed according to the German Law on the Protection of Animals and the corresponding European Communities Council Directive of November 24, 1986 (86/609/EEC), and were approved by the Thüringer Landesamt für Lebensmittelsicherheit und Verbraucherschutz (Thuringia State Office for Food Safety and Consumer Protection) under the registration number 02-015/06.

\subsection{Visual acuity and contrast sensitivity}

\subsubsection{Virtual-reality optomotor system}

Visual acuity was assessed using the recently developed virtual-reality optomotor system (Prusky et al., 2004). The setup consists of a box made of four computer screens onto which the stimuli are projected, with ceiling and floor made of mirrors. The animals are observed via a video camera in the top lid of the box. Freely moving animals are exposed to moving sine wave gratings of various spatial frequencies and contrasts and will reflexively track the gratings by head movements as long as they can see the gratings. Spatial frequency at full contrast and contrast at six different spatial frequencies were varied by the experimenter until the threshold of tracking was determined. Contrast sensitivity thresholds measured in percent were converted into Michelson contrasts based on screen luminance $(\max -\min ) /(\max +\min )$ by taking the reciprocal of the threshold, which was multiplied by a factor of 0.985 for the proportion of minimal and maximal luminances: e.g., 25\% contrast are thus expressed as 4.06 in absolute contrast values. Percent values are mentioned in the results section in addition to the absolute values for better comprehensibility.

\subsubsection{Visual water task}

As a second method to assess visual acuity, we used the visual water task, a visual discrimination task based on reinforcement learning (Prusky et al., 2000; Prusky and Douglas, 2004). In this task, animals were initially trained to distinguish a low spatial frequency vertical grating $(0.086 \mathrm{cyc} / \mathrm{deg})$ from equiluminant grey, before their ability to recognize higher spatial frequencies was tested. The apparatus consists of a trapezoidalshaped pool with two monitors placed side by side at one end. A midline divider is extended from the wide end into the pool, creating a maze with a stem and two arms. The length of the divider sets the choice point between the two visual stimuli. An escape platform that is invisible to the animals is placed below the monitor showing the grating. The position of the grating and platform were alternated in a pseudorandom sequence over the training and test trials. Once $90 \%$ accuracy was achieved, the discrimination threshold 
was determined by increasing the spatial frequency of the grating until performance fell below $70 \%$ accuracy. The highest spatial frequency at which $70 \%$ accuracy was achieved was taken as the maximum visual acuity. The entire task was performed with the room lights off.

\subsection{Interocular plasticity}

For probing visual plasticity, we monocularly deprived mice according to published protocols (Gordon and Stryker, 1996). In all cases, the right eyes were sutured shut. Animals were checked daily to make sure that the eyes remained closed; animals in which the eye was not completely closed throughout the deprivation period were excluded from further experiments. As recently reported, MD induced a cortex-dependent enhancement of the optokinetic response of the non-deprived eye after daily testing in adult mice (Prusky et al., 2006). To analyze this "interocular plasticity" in ageing mice, both visual acuity and contrast sensitivity of the open eye were measured daily for seven days in the virtual-reality optomotor system and the same animals were subjected to an optical imaging experiment immediately after the behavioural measurements to visualize visual cortical activity and plasticity (see below).

\subsection{Optical imaging}

After initial anaesthesia with $2 \%$ halothane in $1: 1 \mathrm{O}_{2} / \mathrm{N}_{2} \mathrm{O}$ mixture, the animals received an intraperitoneal injection of $50 \mathrm{mg} / \mathrm{kg}$ pentobarbital, supplemented by chlorprothixene $(0.2 \mathrm{mg} / \mathrm{mouse}$, i.m.), atropine $(0.3 \mathrm{mg} / \mathrm{mouse}$, s.c.) and dexamethasone $(0.2 \mathrm{mg} / \mathrm{mouse}$, s.c.). A tracheotomy was performed and the animals were placed in a stereotaxic apparatus. In addition, lidocaine (2\% xylocaine jelly) was applied locally to all incisions. Body temperature was maintained at $37^{\circ} \mathrm{C}$ and the electrocardiogram was monitored throughout the experiment. Anaesthesia was maintained with $0.6-0.8 \%$ halothane in a mixture of $1: 1 \mathrm{O}_{2} / \mathrm{N}_{2} \mathrm{O}$ applied through the tracheal tube. A craniotomy was prepared over the left hemisphere. The exposed area was covered by agarose $(2.5 \%)$ and a glass coverslip.

Mouse visual cortical responses were recorded using the imaging method of temporally encoded maps (Kalatsky and Stryker, 2003) and optimized for the assessment of ocular dominance plasticity by Cang and colleagues (Cang et al., 2005a). Briefly, optical images of cortical intrinsic signals were obtained using a Dalsa 1M30 CCD camera (Dalsa, Waterloo, Canada) controlled by custom software. Using a $135 \times 50 \mathrm{~mm}$ tandem lens configuration (Nikon, Inc., Melville, NY), we imaged a cortical area of $4.6 \times 4.6 \mathrm{~mm}^{2}$. The surface vascular pattern and intrinsic signal images were visualized with illumination wavelengths set by a green $(550 \pm 3 \mathrm{~nm})$ or red $(610 \pm 3 \mathrm{~nm})$ interference filter, respectively. Frames were acquired at a rate of $30 \mathrm{~Hz}$, temporally binned to $7.5 \mathrm{~Hz}$ and stored as $512 \times 512$ pixel images after spatial binning of the camera image.

\subsubsection{Visual stimuli}

A high refresh rate monitor (Hitachi Accuvue HM 4921-D) was used to display the visual stimuli: a horizontal bar drifted up and down to reveal elevation maps and a vertical bar drifted left and right to reveal azimuth maps. Drifting horizontal or vertical bars were generated by a Matrox G450 board (Matrox Graphics ${ }^{\circledR}$, Inc., Quebec, Canada), controlled by custom software. The drifting bars were $2^{\circ}$ wide, corresponding to a spatial frequency of $0.25 \mathrm{cyc} / \mathrm{deg}$. The distance between two bars was $80^{\circ}$ and they were presented at a temporal frequency of $0.125 \mathrm{~Hz}$.

To quantify maximum response strength (visual cortical activity) and the quality of retinotopic maps, the monitor was placed in the right visual field of the animal at a distance of $25 \mathrm{~cm}$ to optimally stimulate the right eye (contralateral to the recorded hemisphere). The visual stimuli covered $79^{\circ}$ azimuth and $58^{\circ}$ elevation. For the assessment of ocular dominance, the monitor was positioned in the binocular visual field of the recorded left hemisphere $\left(-5^{\circ}\right.$ to $+15^{\circ}$ azimuth $)$ in front of the animal and visual stimuli were presented alternately to the left and right eye.

\subsubsection{Data analysis}

Elevation and azimuth maps of the contralateral visual field were calculated from the acquired frames by Fourier analysis to extract the signal at the stimulation frequency using custom software as described previously (Kalatsky and Stryker, 2003; Lehmann \& Löwel, 2008). While the phase component of the signal is used for the calculation of retinotopy, the amplitude component represents the intensity of neuronal activation (expressed as fractional change in reflectance $\times 10^{4}$ ). To assess the quality of retinotopic maps, we used the calculation introduced by Cang et al. (2005b). Briefly, both the elevation and azimuth maps were used 
to select the most responsive 20,000 pixels $\left(1.60 \mathrm{~mm}^{2}\right.$ of cortical space) in the visual cortex. For each of these pixels, the difference between its phase and the mean phase of its surrounding 24 pixels was calculated. For maps of high quality, the position differences are quite small because of smooth progression. The standard deviation of the position difference was then used as an index of the quality of retinotopic maps with small values indicating high map quality and high values indicating low map quality (for details see Cang et al., 2005b).

Ocular dominance indices (ODIs) were calculated as described previously (Cang et al., 2005a; Lehmann and Löwel, 2008). Briefly, activity maps were thresholded at $30 \%$ of peak amplitude, and OD was calculated for each pixel in the binocularly responsive region as $(\mathrm{C}-\mathrm{I}) /(\mathrm{C}+\mathrm{I})$, with $\mathrm{C}$ and $\mathrm{I}$ representing the raw response magnitudes of each pixel to stimulation of the contralateral and ipsilateral eye, respectively, and averaged across all selected pixels. We calculated ODIs from blocks of four runs in which the averaged map for each eye had at least a response magnitude of $1 \times 10^{-4}$. Typically, we obtained at least five ODIs per animal; experiments with less than three ODIs were discarded from further analyses. The ODIs of one animal were averaged for statistical comparisons.

\subsection{Statistical analyses}

Groups were compared by one-way ANOVA. For the behavioural data, days after monocular deprivation and spatial frequencies were treated as repeated measurement factors. Post-hoc tests were carried out with Bonferroni correction. Ocular dominance indices were analyzed by F-tests, checking for equal distribution, followed by $t$-tests. The levels of significance were set as *: $p<0.05 ; * *: p<0.01 ; * * *: p<0.001$. All data are represented as means \pm s.e.m.

\section{Results}

\subsection{Visual performance as determined with the virtual-reality optomotor setup: Vision declined during ageing in mice}

We investigated visual performance of C57BL/6 mice from 4 to 26 months of age in the virtual-reality optomotor system developed by Prusky and colleagues (2004).

Visual acuity. At group level, visual acuity values of the left and right eyes developed similarly with age (Fig. 1A), were not different between the two eyes at any age $(p>0.18, t$-test $)$ and were therefore averaged for display. In contrast, in individual animals, the differences between the left and the right eye increased with age, which will be analysed in more detail later (3.5). In mice between 117 and 541 days (4 to 18 months) of age, visual acuity was quite stable at $0.38-0.39 \mathrm{cyc} / \mathrm{deg}$, with little interindividual variance. In older animals from 592 to 824 days (20 to 27 months) of age, visual acuity started to decline and interindividual variance increased. In some of the oldest animals, visual acuity declined to 0.17 cyc/deg. The correlation of age and visual acuity was highly significant $(r=-0.74, p<0.001)$. We further analysed the acuity values in the age groups defined in the methods section (Fig. 1B). In 4 month-old (4M) mice, visual acuity was $0.39 \pm 0.003 \mathrm{cyc} / \mathrm{deg}$ in each of the two eyes $(n=10)$. This value remained stable until approximately one year of age (12M) and then declined steadily to $0.34 \pm 0.007 \mathrm{cyc} / \mathrm{deg}$ in the left and $0.34 \pm 0.005 \mathrm{cyc} / \mathrm{deg}$ in the right eye $(n=32)$ in two-year-old $(23 \mathrm{M})$ mice, followed by a more drastic decline to $0.26 \pm 0.011 \mathrm{cyc} / \mathrm{deg}$ (left eye) and $0.27 \pm 0.01$ (right eye) in 26-month-old $(26 \mathrm{M})$ mice $(n=15)$, such that visual acuity declined on average by $30 \%$ between $4 \mathrm{M}$ and $26 \mathrm{M}$ mice. Visual acuity values of the intermediate age groups were as follows: $7 \mathrm{M}: 0.39 \pm 0.002 \mathrm{cyc} / \mathrm{deg}(n=31), 12 \mathrm{M}: 0.38 \pm 0.003$ cyc/deg ( $n=10), 18 \mathrm{M}: 0.36 \pm 0.006 \mathrm{cyc} / \mathrm{deg}(n=6)$. In all three age groups, the average values of the left and right eye were identical.

Statistical analyses confirmed that age had a significant influence on visual acuity $\left(\mathrm{F}_{5,98}=52.078\right.$, $p<0.001$, ANOVA). Visual acuity values of $4 \mathrm{M}, 7 \mathrm{M}$ and $12 \mathrm{M}$ animals were significantly higher than those of $23 \mathrm{M}$ animals ( $p<0.001$, Bonferroni post-hoc), and all groups had significantly higher visual acuity values than 26M animals $(p<0.001$, Bonferroni post-hoc; Fig. 1A, B). In contrast, the repeated-measures factor eye (i.e., whether visual acuity was measured in the left or the right eye) had no influence on the data $\left(\mathrm{F}_{1,97}=1.229, p=0.27\right)$.

Contrast sensitivity. Contrast sensitivity was measured at 6 different spatial frequencies and peaked at $0.064 \mathrm{cyc} / \mathrm{deg}$ in all age groups, as described previously (Prusky et al., 2004). It developed similarly in 
A

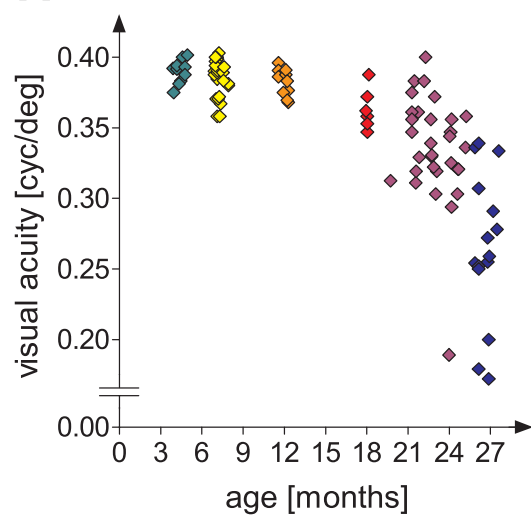

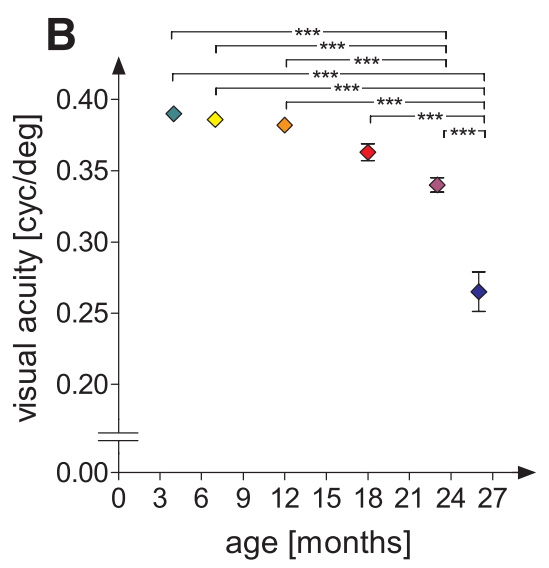

age [months]

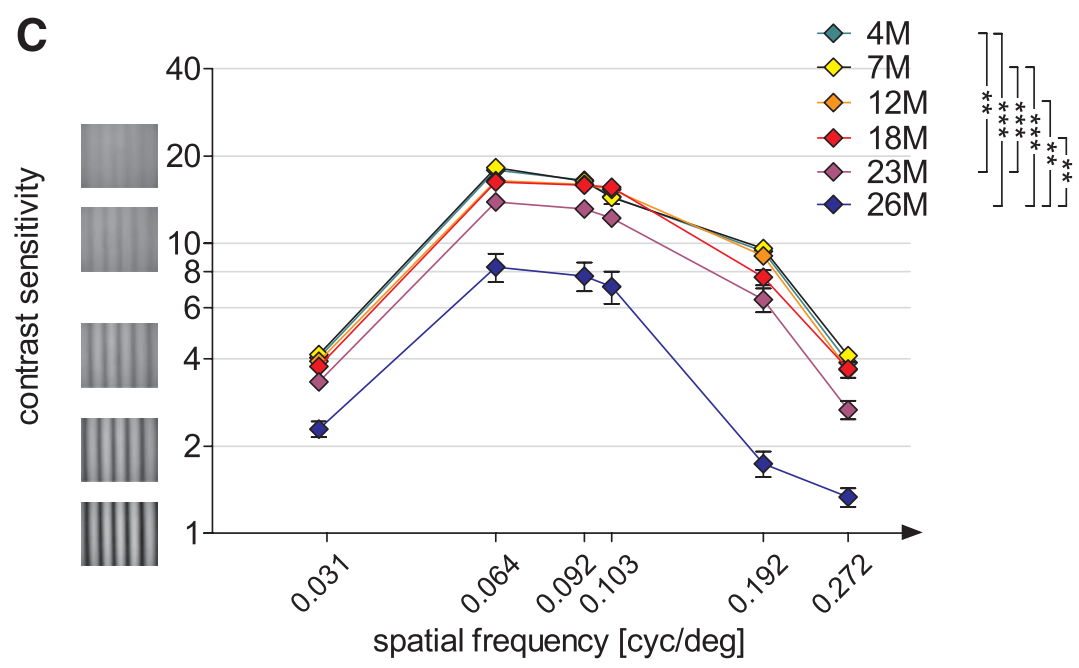

Fig. 1. Visual acuity and contrast sensitivity declined in ageing mice. We used a virtual-reality optomotor system to measure visual acuity and contrast sensitivity of the left and right eye in mice of 117 through 824 days of age. As the values were not different at group level between the two eyes at any age ( $p>0.18, t$-test), mean values were displayed. (A) Visual acuity as a function of age in individual C57BL/6 mice. Visual acuity was 0.38 to $0.39 \mathrm{cyc} / \mathrm{deg}$ in mice up to 541 days (about 18 months), and then started to decline drastically in mice older than 750 days (about 26 months). Note that the y-axis is truncated. (B) Visual acuity plotted as a function of age in the six age groups of the present study (4M, 7M, 12M, 18M, 23M, 26M). Visual acuity was approximately $0.39 \mathrm{cyc} / \mathrm{deg}$ in mice up to one year of age and then declined to 0.34 cyc/deg in $23 \mathrm{M}$ and $0.27 \mathrm{cyc} / \mathrm{deg} 26 \mathrm{M}$ animals $(p<0.001$ in $4 \mathrm{M}$ through $12 \mathrm{M}$ vs. $23 \mathrm{M}$, and $4 \mathrm{M}$ through $23 \mathrm{M}$ vs. $26 \mathrm{M})$. Note that the $\mathrm{y}$-axis is truncated. (C) Contrast sensitivity plotted as a function of spatial frequency in cycles per degree (cyc/deg). Contrast sensitivity declined with age: In $4 \mathrm{M}$ to $18 \mathrm{M}$ animals, contrast sensitivity was approx. $16-18(\approx 6 \%$ contrast $)$ at 0.064 cyc/deg and $4(\approx 25-28 \%)$ at the lowest $(0.031$ cyc/deg) and highest $(0.272 \mathrm{cyc} / \mathrm{deg})$ spatial frequency measured. In $23 \mathrm{M}$ animals, contrast sensitivity declined to $14(\approx 8 \%)$ at $0.064 \mathrm{cyc} / \mathrm{deg}$ and to approximately $3(\approx 33-45 \%)$ at $0.031 \mathrm{cyc} / \mathrm{deg}$ and $0.272 \mathrm{cyc} / \mathrm{deg}$. In $26 \mathrm{M}$ animals, contrast sensitivity declined further to $8(\approx 15 \%)$ at $0.064 \mathrm{cyc} / \mathrm{deg}$, to $2(\approx 50 \%)$ at $0.031 \mathrm{cyc} / \mathrm{deg}$ and to $1(\approx 80 \%)$ at $0.272 \mathrm{cyc} / \mathrm{deg}$. In $23 \mathrm{M}$ animals, contrast sensitivity was significantly lower than in $4 \mathrm{M}$ and $7 \mathrm{M}$ mice $(p<0.01$ and $p<0.001$, respectively); in $26 \mathrm{M}$ mice, it was reduced compared to $4 \mathrm{M}, 7 \mathrm{M}$ (both $p<0.001$ ), 12M and $18 \mathrm{M}$ mice $(p<0.01)$. $* * p<0.01 ; * * * p<0.001$.

both eyes (Fig. 1C). At an age of 4M and 7M, mice still tracked the moving gratings up to a contrast of 18 (corresponding to $5.7 \%$, Fig. 1C) In 23M animals, contrast sensitivity started to decline at all spatial frequencies tested, reaching a peak sensitivity of only $14(=8 \%)$ at $0.064 \mathrm{cyc} / \mathrm{deg}$, which corresponds to a decline of $24 \%$ at this spatial frequency. Across all measured frequencies, we observed an average decline by approximately $23 \%$ as compared to $4 \mathrm{M}$ animals. The contrast curve was shifted to even lower values in $26 \mathrm{M}$ animals, reaching a contrast of only $8(=15 \%)$ at $0.064 \mathrm{cyc} / \mathrm{deg}$, corresponding to an average reduction of $60 \%$ com- 
pared to $4 \mathrm{M}$ animals. Statistical analysis confirmed a significant influence of age on contrast sensitivity (ANOVA, $\mathrm{F}_{5,83}=11.457, p<0.001$ ): Contrast sensitivity of $4 \mathrm{M}$ animals was significantly higher than that of $23 \mathrm{M}(p<0.01)$ and $26 \mathrm{M}$ animals $(p<0.001)$. Values of $7 \mathrm{M}$ animals differed from those of both $23 \mathrm{M}$ and $26 \mathrm{M}$ mice at $p<0.001$, and both $12 \mathrm{M}$ and $18 \mathrm{M}$ mice had significantly better contrast sensitivity than 26M animals $(p<0.01)$ (Fig. 1C).

\subsection{Longitudinal development of visual acuity as determined by both optomotry and the visual water task (Fig. 2)}

The virtual-reality optomotor system measures visual acuity using the optomotor reflex, and in young adult mice, measured values were about $0.2 \mathrm{cyc} / \mathrm{deg}$ lower than when assessed using the visual water task (VWT; e.g., Goetze et al., 2010) which is based on visual discrimination learning and involves the visual cortex. To determine the maximal visual acuity at a given age, we therefore additionally followed visual acuity longitudinally from 12 to 27 months of age in a group of ten mice using both optomotry and the VWT. Since some animals died of decrepitude during the course of the experiment, our sample size decreased from 10 animals at the age of 18 months to 4 animals at 27 months (21 months: 8, 24 months: 5, see Table 2). The animals' physical condition, however, appeared to be good throughout the test. Their fur was glossy and thus well-groomed and they showed no apparent injuries or loss of hair. Concerning locomotor activity, their mean median latency to reach the platform in the VWT was approximately $17 \mathrm{sec}$ during the first test at 12 months and then remained stable at approximately $13 \mathrm{sec}$ until 27 months. According to ANOVA, it was not influenced by age $\left(\mathrm{F}_{4,33}=0.712\right.$, $p>0.5)$. Death never occurred during testing, but always during the intervals in the animals' home cages.

Virtual-reality optomotor system. In the mice analyzed with both tests, reflexive visual acuity was $0.38 \pm 0.001 \mathrm{cyc} / \mathrm{deg}$ in 12 months-old animals and decreased to $0.33 \pm 0.01 \mathrm{cyc} / \mathrm{deg}$ when the animals were 27 months old (Fig. 2A, Table 2). Visual acuity values at intermediate time points were: $0.38 \pm 0.001 \mathrm{cyc} / \mathrm{deg}$ ( 15 months), $0.36 \pm 0.002$ cyc/deg (18 months), $0.35 \pm 0.003 \mathrm{cyc} / \mathrm{deg}$ (21 months), $0.33 \pm 0.008 \mathrm{cyc} / \mathrm{deg}$ (24 months). The decline in visual acuity was thus $14 \%$ between 12 and 27 months of age. ANOVA showed the age-effect to be highly significant $(p<0.001)$. Visual acuity at each time point was significantly different from the values at all but the immediately neighbouring time points $(p<0.05$, Bonferroni-corrected post-hoc). Up to the age of two years ( 24 months), the visual acuity values measured longitudinally in the virtual-reality optomotor system were basically identical to those observed in our cross-sectional analyses (see 3.1), and there was no significant difference (12M: $p=0.25$, 18M: $p=0.81,23 \mathrm{M}$ compared to 24 months: $p=0.67$, $t$-test). Two-way ANOVA, however, detected significant effects both of study (longitudinal vs. cross-sectional; $\left.\mathrm{F}_{1,110}=4.011, p<0.05\right)$ and of interaction between study and age $\left(\mathrm{F}_{3,108}=5.148, p<0.01\right)$. This statistic effect is most probably due to the much more dramatic decline of visual acuity at $26 \mathrm{M}$ in the cross-sectional study $(-30 \%)$ compared to the longitudinal study at 27 months $(-14 \% ; p<0.05$, $t$-test).

$V W T$. Interestingly, cortex-dependent visual acuity followed a different time-course (Fig. 2, Table 2). At 12 months of age, animals had a visual acuity of $0.48 \pm 0.01 \mathrm{cyc} / \mathrm{deg}$, which is close to the value of $0.49 \mathrm{cyc} / \mathrm{deg}$ measured previously in three months old mice (Prusky et al., 2000). At 15 months, this value significantly increased to $0.56 \pm 0.02 \mathrm{cyc} / \mathrm{deg}$. This unexpected increase might be due to continued learning during the first months of the experiment. For the next six months, i.e. up to 21 months of age, visual acuity values remained stable at $0.58 \pm 0.02$ cyc/deg. Thereafter, visual acuity steadily declined to $0.52 \pm 0.02 \mathrm{cyc} / \mathrm{deg}$ at 24 months and to $0.48 \pm 0.03$ cyc/deg at 27 months of age. Age had a significant influence on this perceptual visual acuity (ANOVA, $p<0.01)$. The decline was significant between 21 and 27 months of age $(p<0.05$, Bonferroni-corrected posthoc), and amounted to $19 \% \pm 5 \%$.

The visual acuity values from individual animals (colour-coded) in both tasks are illustrated in Fig. 2B. While values measured in the virtual-reality optomotor system declined in all animals from 12 to 27 months of age, although with varying magnitude, values measured in the VWT showed much more pronounced interindividual variability in both absolute visual acuity values, and time-course of changes during ageing: animals 2, 4 and 7 reached their highest visual acuity values at 21 months of age (animal 2:0.67 cyc/deg; $4: 0.51 \mathrm{cyc} / \mathrm{deg} ; 7: 0.62 \mathrm{cyc} / \mathrm{deg}$ ), when most other animals already showed a decline. 
A

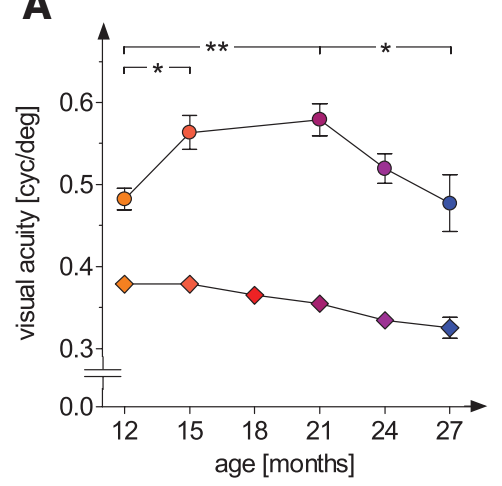

B

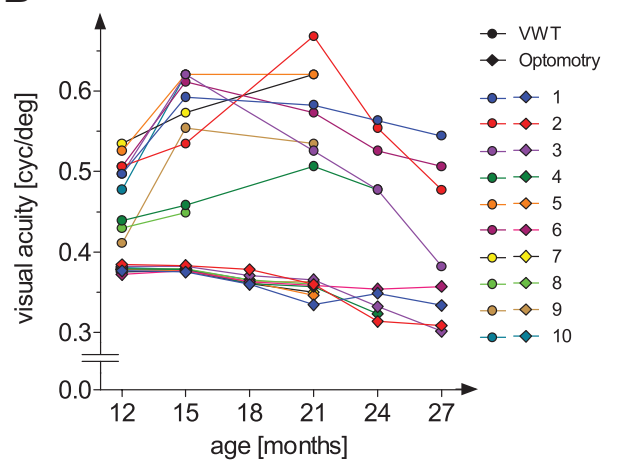

Fig. 2. Longitudinal development of visual acuity in individual mice measured by both optomotry and in the VWT. In a group of ten mice, visual acuity was tested from the age of 12 to 27 months in both the virtual-reality optomotor system (diamonds) and in the visual water task (VWT, circles). (A) Average visual acuity values of the various age groups (colour-coded as in Fig. 1). (B) Colour-coded individual visual acuity values. $* p<0.05 ; * * p<0.01$.

\subsubsection{Comparison of visual performance in the two tests}

The change in visual acuity during ageing shows a different time course between values measured in the visual water task or the virtual-reality optomotor system. ANOVA confirmed a highly significant interaction of method and age $\left(\mathrm{F}_{4,33}=6,038\right.$, $p<0.01)$. In the visual water task, visual acuity reached peak values of approximately 0.56 cyc/deg between 15 and 21 months of age and only started to decline at 24 months, whereas in the virtual-reality optomotor system, visual acuity values declined steadily from 12 months onwards. As expected and already observed in previous studies (Douglas et al., 2005; Goetze et al., 2010), the method chosen to measure visual acuity also had a profound impact on the height of the values $\left(\mathrm{F}_{1,36}=312,135, p<0.001\right)$, with VWT values being constantly much higher than values measured in the virtual-reality optomotor system. The average difference was between $0.10 \mathrm{cyc} / \mathrm{deg}$ at 12 months and $0.22 \mathrm{cyc} / \mathrm{deg}$ at 21 months of age. At 27 months, when VWT values had already markedly declined, average acuity was $0.15 \mathrm{cyc} / \mathrm{deg}$ above the optomotry values.

While interindividual variance in the optomotry values increased steadily from $0.01 \mathrm{cyc} / \mathrm{deg}$ at twelve months to $0.06 \mathrm{cyc} / \mathrm{deg}$ at 27 months, it remained stable over time at approximatley $0.16 \mathrm{cyc} / \mathrm{deg}$ and thus was much higher (3-12 times) for visual acuity values measured in the VWT. When we compared visual acuity values of individual mice obtained by the two methods (Fig. 2B), they were not significantly correlated at any time point. In both behavioural tasks, there was a large interindividual variance in the visual acuity decline between 21 and 27 months of age: Two animals (2 and 3) lost $14 \%$ and $17 \%$ in visual acuity in the virtual-reality optomotor system and 27-29\% in the VWT, while the decline was below 1\% (optomotry) and merely $7 \%$ and $12 \%$ (VWT) in the other two animals (1 and 6). Interestingly, these values were significantly correlated between the two tasks in these four animals $(p<0.05)$, indicating that there is a common cause for the visual acuity decline measured in both methods.

\subsection{Interocular plasticity declined during ageing in mice}

After 7 days of MD and daily testing in the optomotor setup, visual acuity of the open eye increased from $0.39 \pm 0.003 \mathrm{cyc} / \mathrm{deg}$ to $0.51 \pm 0.005 \mathrm{cyc} / \mathrm{deg}$ $(n=7)$ and $0.50 \pm 0.003 \mathrm{cyc} / \mathrm{deg}(n=12)$ in $4 \mathrm{M}$ and $7 \mathrm{M}$ animals, respectively (Fig. 3A, top panel), corresponding to an improvement (gain on baseline) of $29.8 \pm 1.3 \%$ and $28.6 \pm 1.1 \%$ (Fig. 3B, top panel). In contrast, in $23 \mathrm{M}$ animals, visual acuity increased from $0.33 \pm 0.015 \mathrm{cyc} / \mathrm{deg}$ to $0.37 \pm 0.02 \mathrm{cyc} / \mathrm{deg}(n=11)$, corresponding to an increase of only $13.4 \pm 2.7 \%$. Both the absolute visual acuity values (Fig. 3A, $\mathrm{F}_{2,27}=29.439 ; p<0.001$, ANOVA with repeated measures) and the relative increases of visual acuity from baseline (Fig. 3B, $F_{2,27}=8.713, p<0.001$, ANOVA) depended highly significantly on age. Bonferroni post- 
hoc testing further showed that while there was no difference between the two younger age groups (4M and $7 \mathrm{M} ; p>0.5$ ), both absolute and relative values of visual acuity in the two younger groups differed highly significantly from those of $23 \mathrm{M}$ animals $(p<0.001$ and $p<0.01$, respectively).

Complementing average values in the different age groups, we additionally plotted values of both visual acuity (Fig. 3A) and relative gain on baseline (Fig. 3B) of individual mice achieved during daily training. This analysis illustrates the heterogeneity of interocular plasticity among individuals that appears to dramatically increase with age. In $4 \mathrm{M}$ animals, the highest acuity differences between individual mice on any day after MD was $0.041 \mathrm{cyc} / \mathrm{deg}$. In $7 \mathrm{M}$ animals, differences already doubled to $0.094 \mathrm{cyc} / \mathrm{deg}$ and further increased to $0.222 \mathrm{cyc} / \mathrm{deg}$ in $23 \mathrm{M}$ animals (to $0.097 \mathrm{cyc} / \mathrm{deg}$ without one outlier that had extremely poor acuity values already before MD). By comparing gain on baseline, the highest differences on any day after $\mathrm{MD}$ was 11 percentage points in $4 \mathrm{M}$ animals, 21 percentage points in $7 \mathrm{M}$ mice and 35 percentage points in $23 \mathrm{M}$ animals. In this latter group, it even occurred in several subjects that visual acuity initially increased slightly, but then declined during further testing, in one animal even to values below baseline (Fig. 3B, bottom figure). On the other hand, two animals aged 648 and 699 days at MD showed interocular plasticity to a similar degree as average $4 \mathrm{M}$ and $7 \mathrm{M}$ mice: their visual acuity increased from 0.322 cyc/deg and $0.344 \mathrm{cyc} / \mathrm{deg}$ to $0.414 \mathrm{cyc} / \mathrm{deg}$ and 0.419 cyc/deg, corresponding to a gain on baseline of $22 \%$ and $29 \%$.

\subsection{Visual cortical activity and retinotopic map quality were reduced in old mice}

To analyze age-related changes in visual cortical activity directly, we visualized both elevation maps after visual stimulation with moving horizontal bars and azimuth maps after stimulation with moving vertical bars (Fig. 4) using optical imaging of intrinsic signals (Kalatsky and Stryker, 2003) in 4M, 7M and $23 \mathrm{M}$ mice. At all ages, visual cortical maps displayed clear and apparently well-organized retinotopy. Quantitative analyses of the optical imaging data revealed that the magnitude of the visual cortical responses significantly depended on age $\left(\mathrm{F}_{2,17}=6.143, p<0.01\right.$, ANOVA). In $4 \mathrm{M}$ and $7 \mathrm{M}$ animals, signal amplitudes were not significantly different $(p=0.69, t$-test $)$. In contrast, there was a significant difference between signal amplitudes in the $7 \mathrm{M}$ and $23 \mathrm{M}$ groups $(p<0.05$, Bonferroni post-hoc): The signal amplitude component of the imaging signal was $2.6 \pm 0.28 \times 10^{-4}$ in $4 \mathrm{M}$ animals $(n=4)$ and significantly decreased from $2.7 \pm 0.23 \times 10^{-4}(n=8)$ in $7 \mathrm{M}$ to $1.8 \pm 0.14 \times 10^{-4}$ $(n=8)$ in $23 \mathrm{M}$ animals, corresponding to a decrease of about $33 \%$ between $7 \mathrm{M}$ and $23 \mathrm{M}$ (Fig. 4, right column).

Similarly, the quality of retinotopic maps (low standard deviation corresponds to low map scatter and thus to high map quality, see methods and Cang et al., $2005 \mathrm{~b})$ decreased with age $\left(\mathrm{F}_{2,17}=4.877, p<0.05\right.$, ANOVA), again with a significant difference between $7 \mathrm{M}$ and $23 \mathrm{M}$ groups $(p<0.05$, Bonferroni post-hoc): for elevation maps, map scatter was $1.1 \pm 0.25$ in $4 \mathrm{M}$ animals $(n=4)$ and $1.0 \pm 0.11$ in $7 \mathrm{M}$ animals $(n=8)$. In $23 \mathrm{M}$ animals $(n=8)$, map scatter was $1.75 \pm 0.21$ (Fig. 4, right column), corresponding to an increase of $39 \%$. It should be noted, however, that map scatter is inversely proportional to the magnitude of the cortical responses. If amplitude was taken as a covariable into statistical analysis, there was no detectable influence of age on map quality $\left(\mathrm{F}_{2,16}=0.973, p=0.4\right)$.

In the optically recorded azimuth maps, signal amplitude was also smaller in $23 \mathrm{M}$ animals $\left(1.67 \pm 0.17 \times 10^{-4}, n=8\right)$ compared to $4 \mathrm{M}(2.04 \pm$ $\left.0.48 \times 10^{-4}, \quad n=3\right)$ and $7 \mathrm{M}$ animals $(2.44 \pm$ $\left.0.23 \times 10^{-4}, n=8\right)$. After pooling values of $4 \mathrm{M}$ and $7 \mathrm{M}$ animals, which were not different from each other $(p>0.4, t$-test), response magnitudes were significantly higher in the two younger age groups compared to $23 \mathrm{M}$ animals ( $p<0.05, t$-test).

Retinotopic scatter of azimuth maps was also increased in $23 \mathrm{M}$ animals $(7.62 \pm 1.26, n=8)$ compared to $4 \mathrm{M}(5.7 \pm 1.13, n=3)$ and $7 \mathrm{M}$ animals $(5.35 \pm 1.54, n=8)$, but this tendency was not statistically significant $\left(\mathrm{F}_{2,16}=0.771, p>0.4\right.$, ANOVA $)$, even if $4 \mathrm{M}$ and $7 \mathrm{M}$ data were pooled $(p>0.2, t$-test).

We have also tested if retinotopic map activity reflected visual acuity on an individual level. As the virtual-reality optomotor system allows to measure the acuity of each eye separately, we correlated visual acuity of the right eye with map amplitudes recorded after stimulation of that eye. Map amplitudes and visual acuity in individual mice were neither significantly correlated for elevation maps ( $r=-0.20$ and -0.16 , respectively, $p>0.5$ ) nor for azimuth maps $(r=0.37$ and -0.16 , respectively, $p>0.4)$. 
A

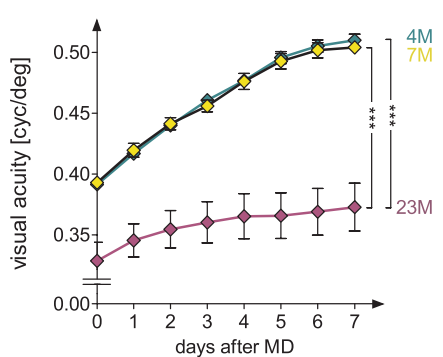

B

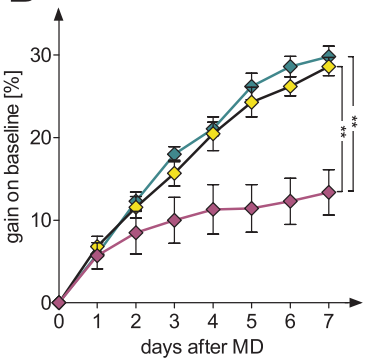

$4 \mathrm{M}$
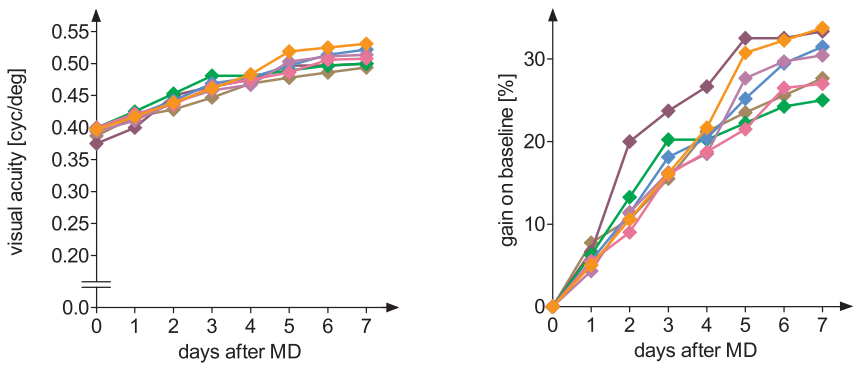

$7 \mathrm{M}$
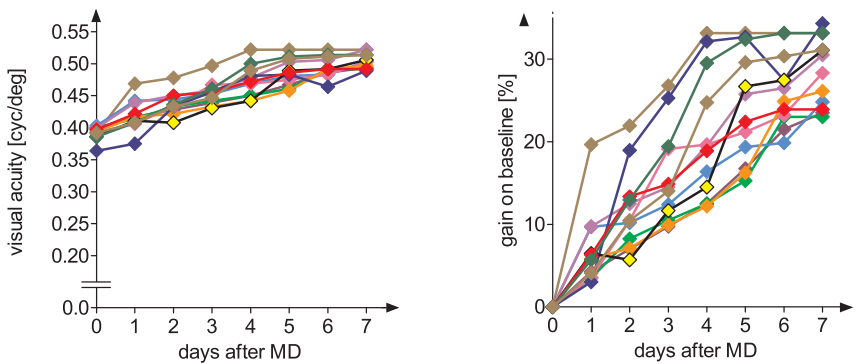

$23 \mathrm{M}$

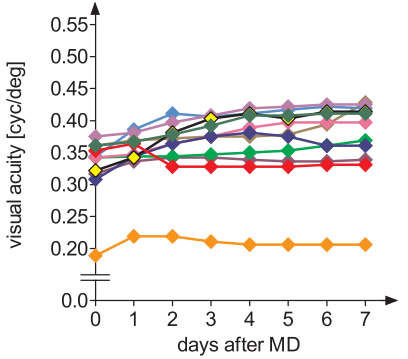

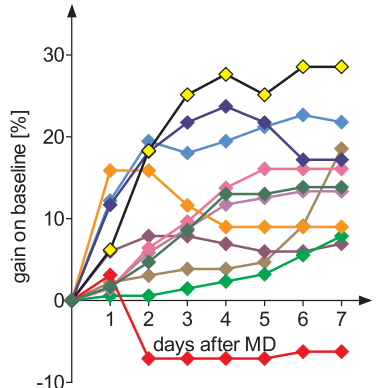

Fig. 3. Interocular plasticity was reduced in old mice. Mice were monocularly deprived and tested daily in the virtual-reality optomotor system. Visual acuity $(\mathrm{A}$, in cycles per degree $=\mathrm{cyc} / \mathrm{deg})$ and gain on baseline $(\mathrm{B}$, in \%) are plotted as a function of days after monocular deprivation (MD). (A) During seven days of MD, visual acuity of the open eye increased in all investigated age groups, but significantly less in $23 \mathrm{M}$ (from $0.33 \mathrm{cyc} / \mathrm{deg}$ on day 1 to $0.37 \mathrm{cyc} / \mathrm{deg}$ on day 7$)$ compared to $4 \mathrm{M}$ and $7 \mathrm{M}$ animals $(0.39 \rightarrow 0.51$ respectively $0.50 \mathrm{cyc} / \mathrm{deg})$. Individual values of mice of the $4 \mathrm{M}, 7 \mathrm{M}$ and $23 \mathrm{M}$ age groups are shown below. Note that interindividual variability strongly increased with age. Note also that the $\mathrm{y}$-axis is truncated. (B) The relative increase of visual acuity after MD (gain on baseline) was significantly higher in $4 \mathrm{M}$ (30\% between days 1 and 7) and $7 \mathrm{M}$ animals (29\%) compared to $23 \mathrm{M}$ animals (13\%). Individual values of mice from the three age groups are illustrated below. Note the pronounced increase of interindividual variability with age. At an age of 23 months, some individuals showed interocular plasticity to a degree similar to that of $4 \mathrm{M}$ and $7 \mathrm{M}$ animals, while others exhibited little plasticity or no gain during the course of training. $* * p<0.01$; $* * * p<0.001$ 

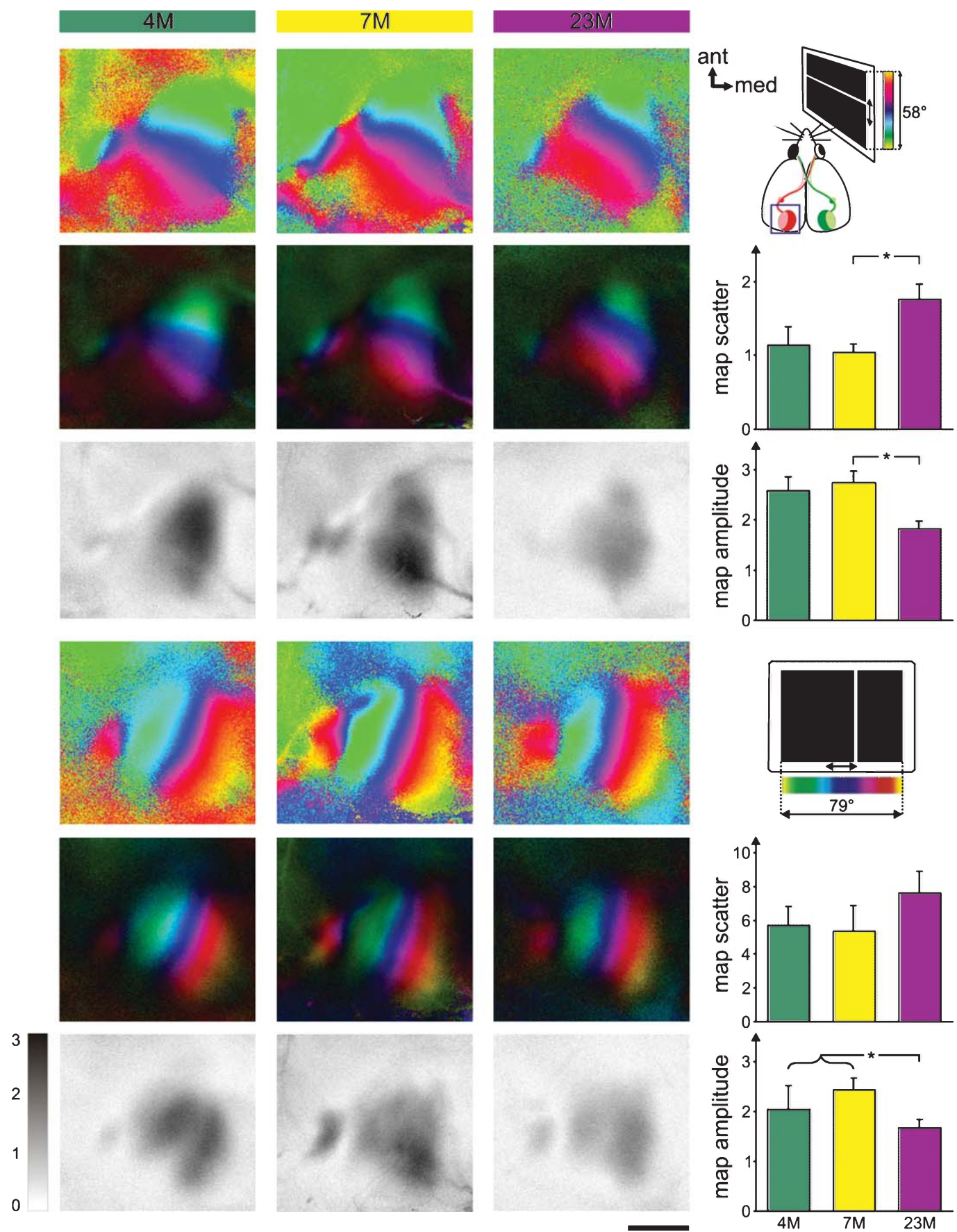

Fig. 4. Decreased activation in the visual cortex of old mice. Cortical activity and retinotopic maps in ageing mice recorded by intrinsic signal optical imaging. Representative elevation (top) and azimuth (bottom) maps of 4M, 7M and 23M animals are displayed. For each age-group and map orientation, phase (top), polar (centre) and response magnitude maps (bottom) are shown. Retinotopy is plotted according to the colour-code shown in the insets in the right column. Response magnitude or map amplitude, expressed as fractional change in reflectance $\times 10^{4}$, is grey-scale coded according to the bar at the left of the figure. Quantifications of map amplitude and map scatter are illustrated in the right column: The amplitude of elevation maps was significantly lower in 23M compared to $7 \mathrm{M}$ animals. Map scatter was significantly increased in these animals. Similarly, the amplitude of azimuth maps was decreasead in $23 \mathrm{M}$ animals compared to the pooled values of $4 \mathrm{M}$ and $7 \mathrm{M}$ animals, but map scatter was not significantly different. The scale bar is $1 \mathrm{~mm}$ and applies to all panels showing maps. $* p<0.05$. 


\subsection{Visual acuity asymmetry and ocular dominance in old mice}

Using optical imaging of intrinsic signals (Kalatsky and Stryker, 2003; Cang et al., 2005a), we also visualized ocular dominance (OD) in $4 \mathrm{M}, 7 \mathrm{M}$ and $23 \mathrm{M}$ mice. Data for the $4 \mathrm{M}$ and $7 \mathrm{M}$ mice were reproduced from (Lehmann and Löwel, 2008). As described previously, the visual cortex of rodents is dominated by input from the contralateral eye: the average OD index (ODI) of all investigated animals was above zero. The mean ODI declined with age from 0.26 in $4 \mathrm{M}$ mice to 0.22 in $7 \mathrm{M}$ and 0.15 in 23M mice (Fig. 5A). Statistical comparison confirmed that OD was influenced by age $\left(\mathrm{F}_{2,29}=4.707, p<0.05\right.$, ANOVA), with a significant difference between the $4 \mathrm{M}$ and $23 \mathrm{M}$ groups $(p<0.05$, Bonferroni post-hoc). This reduction in contralateral dominance was predominantly due to a stronger decrease in cortical activation after stimulation of the contralateral compared to the ipsilateral eye: Map amplitude in binocular V1 after contralateral eye stimulation declined significantly by $26 \%$ ( $p<0.05, t$-test $)$ from $2.3 \pm 0.14 \times 10^{-4}$ in $4 \mathrm{M}$ to $1.7 \pm 0.19 \times 10^{-4}$ in $23 \mathrm{M}$ animals, while the decline was only $15 \%$ from $1.6 \pm 0.11 \times 10^{-4}$ to $1.37 \pm 0.09 \times 10^{-4}(p=0.13)$ after stimulation of the ipsilateral eye.

While we have shown previously that OD-plasticity after MD is absent in mice older than 110 days of age (Lehmann and Löwel, 2008), the oldest mice that were investigated so far in this paradigm were seven months old. We therefore extended this age range and also analyzed 2-year-old animals in the present study. Indeed, seven days of MD failed to change ocular dominance in 23M animals (Fig. 6). Cortical activity elicited by stimulation of the contralateral eye was stronger than after stimulation of the ipsilateral eye both in control animals and after seven days of MD and the 2-dimensional OD-maps showed mostly warm colours indicating a dominance of the contralateral eye. On average, the ODI was $0.15 \pm 0.05$ in control animals $(n=9)$ and $0.17 \pm 0.05$ after seven days of MD ( $n=7$; Fig. 5A). These values were not significantly different $(p>0.5$, $t$-test).

Interestingly, the variance of ODI-values dramatically increased between $4 \mathrm{M}$ and $23 \mathrm{M}$ animals: while the maximal difference in ODI-values was 0.14 in $4 \mathrm{M}$ animals, it almost doubled to 0.24 in $7 \mathrm{M}$ mice and tripled to 0.38 in $23 \mathrm{M}$ mice, thus spanning nearly the entire range of observed ODI-values irrespective of the experimental condition in the older age group. To test whether the high variance reflected intraindividual asymmetry in the visual acuities of the two eyes we compared these values in individual animals and indeed found that differences between the acuities of the two eyes significantly increased with age (Fig. 7A; $r=0.334, p<0.001)$ : Up to the age of 20 months, the differences were scattered near the $\mathrm{x}$-axis, indicating that in each individual animal, both eyes had similar acuities. The highest differences in visual acuity between the two eyes were $0.006 \mathrm{cyc} / \mathrm{deg}$ in $4 \mathrm{M}$ mice, $0.009 \mathrm{cyc} / \mathrm{deg}$ in $7 \mathrm{M}$ and $12 \mathrm{M}$ mice, $0.003 \mathrm{cyc} / \mathrm{deg}$ in $18 \mathrm{M}$ mice. In contrast, in animals older than 20 months, the highest acuity differences increased to $0.186 \mathrm{cyc} / \mathrm{deg}$ in $23 \mathrm{M}$ and to $0.072 \mathrm{cyc} / \mathrm{deg}$ in $26 \mathrm{M}$ mice. It is worth noting - however - that the majority $(53 \%$ of $23 \mathrm{M}, 66 \%$ in $26 \mathrm{M})$ of individual old mice still had acuity differences that were lower than 0.009 cyc/deg, i.e. the highest difference found in younger animals. Interestingly, standard errors of the visual acuity differences were also significantly correlated with age (Fig. 7B, $r=0.851, p<0.05$ ). Taken together, our analyses indicate that visual acuity values in the two eyes of old mice were significantly less similar compared to younger animals, with most old animals still having almost equal vision in both eyes, while others had much worse vision in one eye than in the other.

To test the assumption that this asymmetry in visual acuities was related to OD-indices in $23 \mathrm{M}$ mice, we calculated a visual acuity asymmetry index (VAAI) of right and left eye visual acuities for each animal in a similar fashion as the ODI, i.e. (rightleft)/(right + left): positive VAAI-values thus indicate a higher visual acuity of the right eye. In $23 \mathrm{M}$ control animals, there was a weak correlation between the ODI and the VAAI that was - however - not statistically significant (Fig. 5B, $r=0.736, p=0.059$ ). Nevertheless, this correlation suggested that on an individual level, weaker acuity of one eye went along with reduced cortical activity elicited by visual stimulation of that eye. No such relationship was observed in $4 \mathrm{M}$ and $7 \mathrm{M}$ animals $(p>0.8)$.

\subsection{Increased interindividual variability in ageing mice}

A major observation of our analyses was that interindividual variability increased with age in some of the measured parameters. To test this impression quantitatively we correlated age with the standard 

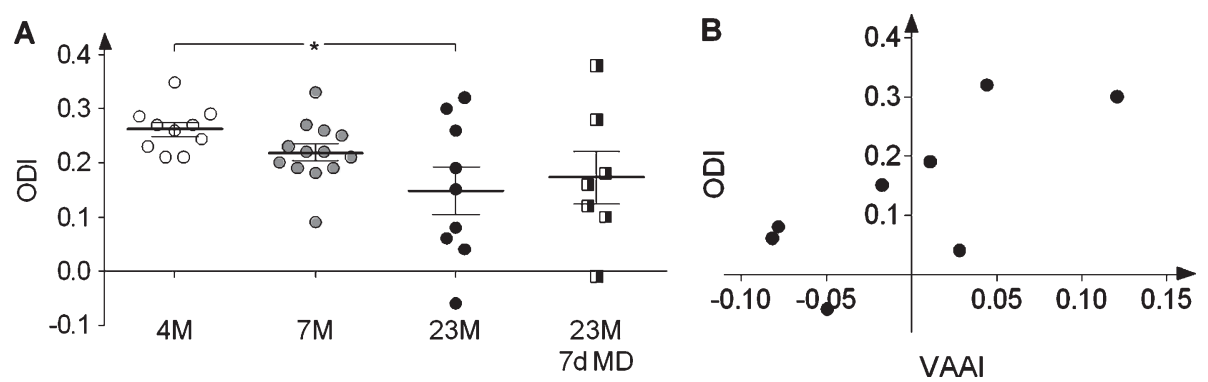

Fig. 5. Reduced ocular dominance in 23M mice. Quantification of ocular dominance (OD) from optically imaged visual cortical maps of $4 \mathrm{M}$, $7 \mathrm{M}$ and 23M old mice. A positive OD-index (ODI) indicates dominance of the contralateral eye, a negative ODI ipsilateral dominance. Symbols represent ODI-values of individual animals, means are marked by the thick horizontal lines. (A) In 4M animals, the mean ODI was $0.26,0.22$ in $7 \mathrm{M}$ mice and then declined significantly to 0.15 in $23 \mathrm{M}$ mice $(p<0.05$, Bonferroni post-hoc). Monocular deprivation (MD) for seven days in $23 \mathrm{M}$ mice did not shift ODI values (0.17). Note the pronounced interindividual variability of ODI-values in 23M mice. (B) ODIs of individual $23 \mathrm{M}$ mice plotted as a function of their individual visual acuity asymmetry indices (VAAI). Asymmetry indices were calculated from visual acuity values of both eyes in individual animals. Note that there is a strong tendency for animals in which the right eye had a higher visual acuity compared to the left eye (positive VAAI) to also have a higher ODI, and vice versa. ${ }^{*} p<0.05$.

errors of each group and parameter. In the data obtained in the virtual-reality optomotor system, the standard errors of visual acuity had a strong trend-like correlation with age $(r=0.805, p=0.053)$, underpinning the observation that some $23 \mathrm{M}$ and $26 \mathrm{M}$ animals had visual acuities similar to the mean values of younger groups, while others showed a dramatic deterioration. However, this vision loss did not affect both eyes equally (Fig. 7A), and the standard error of the differences between visual acuities of the two eyes of individual animals was correlated with age $(r=0.851$, $p<0.05$, Fig. 7B). Thus visual acuities of the two eyes became more different with age. Correlations for the standard errors of contrast sensitivities with age were always positive, but never significant $(p>0.2)$. The same held true for visual acuities and relative gain on baseline on the seventh day after MD $(r=0.973$, $p=0.147$ and $p=0.975, p=0.143$ ).

In the optical imaging data, there was no correlation of the standard errors of map amplitudes nor map scatter with age. In contrast, age and the standard errors of ODI-values in 4M, 7M and 23M mice were significantly correlated $(r=0.999, p<0.05)$.

\section{Discussion}

In the present study, we have shown that both visual acuity and contrast sensitivity significantly declined during ageing in mice, as did interocular plasticity after monocular deprivation (MD) as measured using the optomotor response. Visual acuity measured in the cortex-dependent visual water task initially increased with continued training and then also decreased with age. In addition, in old mice, visual cortical activation was markedly reduced in $23 \mathrm{M}$ compared to $7 \mathrm{M}$ mice. Ocular dominance also decreased with age and displayed extreme interindividual variance in $23 \mathrm{M}$ animals, which might be related to increasing differences in visual acuity between the two eyes. Interindividual variability generally increased with age, but interestingly, some $23 \mathrm{M}$ mice retained visual acuity and interocular plasticity like $4 \mathrm{M}$ and $7 \mathrm{M}$ animals.

\subsection{Visual performance of mice declines in old age}

In the visual water task, average visual acuity was $0.48 \mathrm{cyc} / \mathrm{deg}$ at 12 months, significantly increased to maximal values of about $0.56 \mathrm{cyc} / \mathrm{deg}$ up to an age of 21 months and then declined to $0.48 \mathrm{cyc} / \mathrm{deg}$ at 27 months. This behavioural task involves the visual cortex (Douglas et al., 2005) and values measured using this task probably reflect the perceptual limit of an animal. A previous study using the same experimental setup provided somewhat different results: Visual acuity was $0.48 \mathrm{cyc} / \mathrm{deg}$ in six month old mice, then declined to $0.38 \mathrm{cyc} / \mathrm{deg}$ in one year old mice and remained stable at this value up to the age of two years (Wong and Brown, 2007). The difference is most probably due to the fact that in the cited study, animals merely performed 64 trials at eight spatial frequencies in random order, whereas we typically had animals swim 200-300 trials for each data point, slowly increasing spatial 


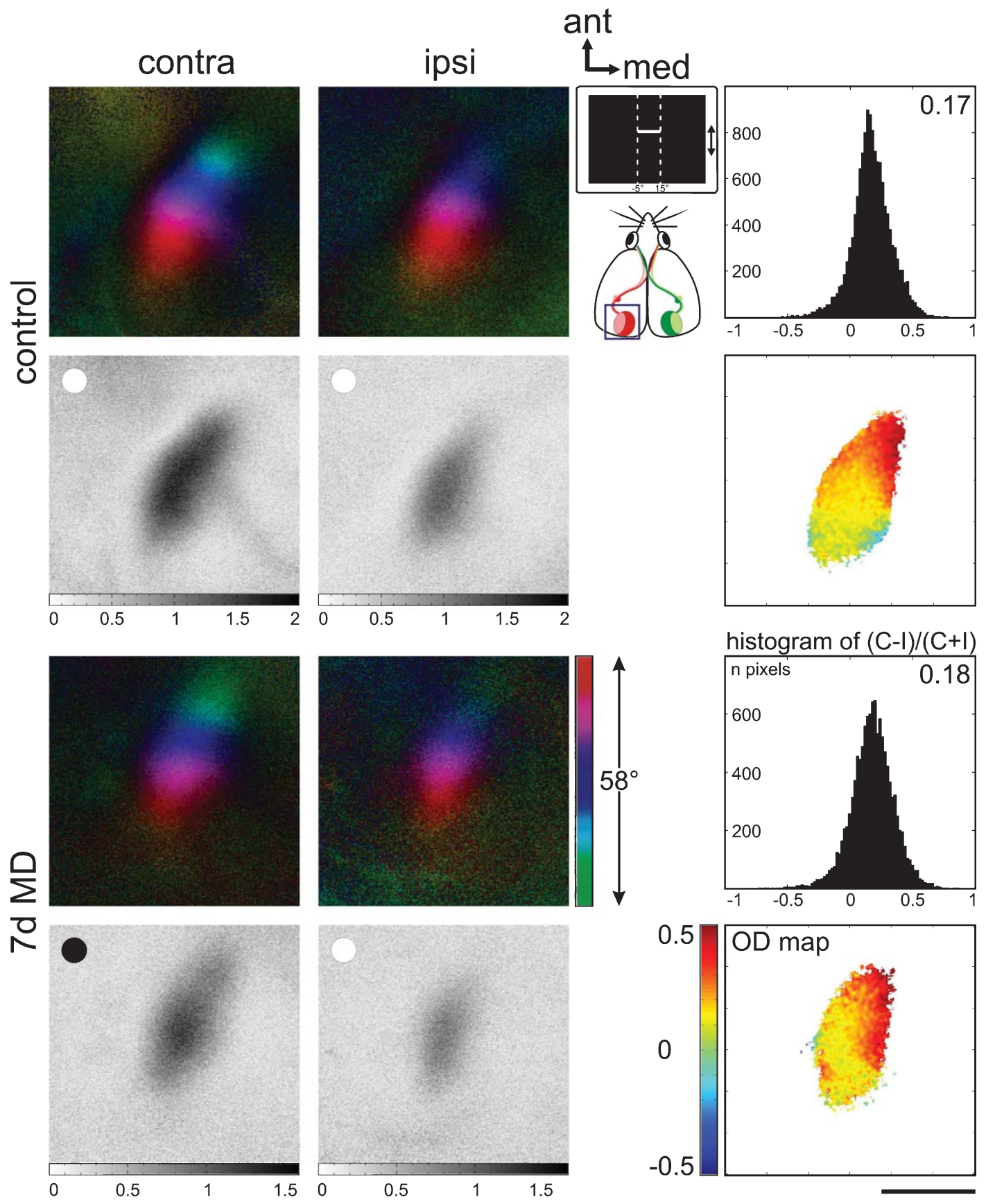

Fig. 6. No ocular dominance plasticity in 23M mice. Optical imaging maps of responses to the ipsi- and contralateral eye in the binocular region of 23M mouse visual cortex in both a control animal (top) and an animal after 7 days of MD (bottom) are shown. Both colour-coded polar maps of retinotopy (top) and grey-scale coded response magnitude maps (below) of the contralateral (contra, left) and ipsilateral (ipsi, right) eye are illustrated. For both experiments, the histogram of ocular dominance (OD) scores, the average OD-index (ODI) and the corresponding 2-D OD-maps (ODI values colour-coded according to the scheme shown at the lower right OD map: blue represents negative, red positive values) are included. Note that in both the control and the 7d MD animal, activity patches evoked by stimulation of the contralateral eye were darker than those after stimulation of the ipsilateral eye and 2-D OD-maps were red and yellow, indicating a contralateral dominance in the binocular region of the visual cortex. In addition, OD-indices were similar in both experimental conditions: 0.17 in control and 0.18 after 7 days of MD. Thus, MD did not induce a significant OD-shift in 23M mice. The scale bar is $1 \mathrm{~mm}$ and applies to all panels showing maps. 

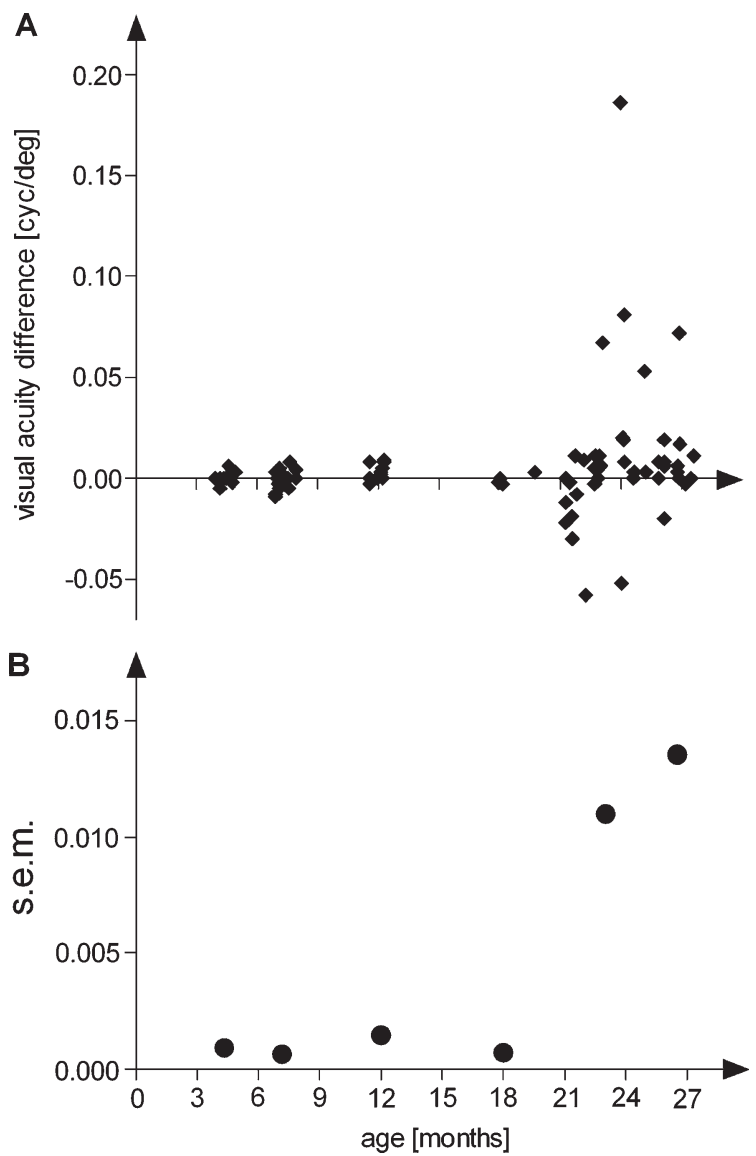

Fig. 7. Visual acuity differences between left and right eyes of individual mice increased with age. (A) For each animal, the difference in the visual acuity of the right and left eye was plotted as a function of age. While visual acuities of the two eyes were rather similar in animals up to 20 months of age, their difference increased significantly thereafter in some mice: in individual animals, either left or right eyes could have better visual acuity. (B) Standard errors for the group analyses, plotted as a function of age, also increased with age.

frequency and thus facilitating learning. Measured in the virtual-reality optomotor system, visual acuity was $0.38-0.39 \mathrm{cyc} / \mathrm{deg}$ in mice up to 18 months of age and then declined to $0.34 \mathrm{cyc} / \mathrm{deg}(-13 \%)$ in mice of 23 months and $0.27 \mathrm{cyc} / \mathrm{deg}(-31 \%)$ in animals of 26 months. This age-dependent decline was significantly weaker (27 months: $0.33 \mathrm{cyc} / \mathrm{deg},-14 \%$ ) in animals that were tested longitudinally in both the optomotor system and the visual water task. This observation suggests that the optomotor response might be stronger or better observable in animals kept fit by swimming on a regular basis. On the other hand, this conclusion is based on only four surviving animals in the longitu- dinal study and needs to be confirmed by additional experiments. Contrast sensitivity also showed a decline that was similar in time-course but even more drastic in degree, dropping on average by $23 \%$ in $23 \mathrm{M}$ and $60 \%$ in $26 \mathrm{M}$ mice. Between mice of four and twelve months, there was no difference in the optokinetic response, which is somewhat at variance with a recent study that found a significant decline in the gain of this reflex already in mice of 9 months, as compared to four months old animals (van Alphen et al., 2009). This discrepancy is probably due to differences in the methods, since the cited study measured eye movements and in general observed much higher contrast sensitivities. In another study, the increment thresholds for both photopic and scotopic vision, which - like contrast sensitivity - require the discrimination of luminances, were unchanged in mice from 89 days up to an age of more than 800 days (Williams and Jacobs, 2007). Discrimination tasks, however, involve the visual cortex, whereas the optokinetic reflex used for measuring vision in the virtual-reality optomotor system is subserved by the subcortical accessory optical system and not changed by cortex ablations (Douglas et al., 2005). In summary, measurements with both methods agree in revealing an age-related decline of mouse visual acuity after around 2 years of age, but the exact timecourse of this decline depends on how acuity is determined. This could reflect a different susceptibility to agerelated changes of the underlying neural systems, with subcortical structures subserving the optomotor reflex deteriorating earlier, compared to the cortical circuits involved in the visual discrimination task.

A dissociation in the age-dependence of two different visual plasticity paradigms has been demonstrated before in the visual cortex, where ocular dominance plasticity ceases after three months of age in mice, while interocular plasticity declines only slightly (Lehmann and Löwel, 2008). Similarly, in the somatosensory cortex of mice, classical conditioninginduced expansion of whisker representation was already absent at 14 months of age while deprivationinduced expansion in layer IV persisted in mice older than two years (Liguz-Lecznar et al., 2009).

\subsection{Age-dependent visual decline may have a cortical component}

We have also analyzed interocular plasticity induced by monocular deprivation (MD), apparent as an increase in visual acuity of the open eye after daily 
testing in the virtual-reality optomotor system (Prusky et al., 2006). In two-year-old mice, interocular plasticity was reduced by more than $50 \%$ compared to four and seven months old animals. The interpretation that this might simply be due to ocular impairments setting an upper limit for the achievable visual acuity is precluded by the fact that much higher visual acuities were observed in animals of similar age in the visual water task. Since interocular plasticity was shown to be mediated by cortical circuits (Prusky et al., 2006), the observed reduction of interocular plasticity in old age indicates that - at least some - age-dependent changes take place at the cortical level.

It is well established that the ocular apparatus deteriorates during ageing: e.g., lens cataracts were found in an increasing proportion of mice and rats older than 18 months (Wolf et al., 2000). In our own data, the age-dependent decline of visual performance observed in the virtual-reality optomotor system is most probably a subcortical phenomenon, given that a reflex was measured. It is also possible that this decline was caused by ocular impairments not detectable by opthalmoscopic inspection. Nevertheless, visual sensitivity, retinal anatomy and physiology do not appear to be causally linked, given that electroretinogram maximal voltages decline in a linear fashion already starting at one month of age (Li et al., 2001; Gresh et al., 2003; Williams and Jacobs, 2007), whereas retinal length, rod and cone density (Trachimowicz et al., 1981; Li et al., 2001), opsin RNA expression and antibody staining (Williams and Jacobs, 2007) remain unchanged even in mice older than two years. The failure to attribute the visual decline observed during ageing solely to the ocular media or the retina has motivated the suggestion that neural visual pathways might be responsible for these changes (Spear, 1993). In monkeys, cats and rats, extracellular single-unit recordings have shown neurons in the visual cortex of aged animals to display lower orientation and direction selectivity and reduced signal-to-noise ratio than in young animals (Schmolesky et al., 2000; Hua et al., 2006; Wang et al., 2006). Neuron number is reduced in the visual cortex of aged rats (Yates et al., 2008). If similar changes in cortical anatomy and function occur in aged mice, they might explain the increase in retinotopic map scatter that we have observed in old animals in the present study. Similarly, age-related impairments in sensory processing have been found in the rat auditory and somatosensory cortices (Mendelson and Ricketts, 2001; Godde et al., 2002; David-Jürgens et al., 2008).
In line with these results, our findings (i) that visual cortical activity is reduced by one third in $23 \mathrm{M}$ animals compared to young adult animals, (ii) that visual acuity measured in the VWT is reduced by $19 \%$ between 21 and 27 months, (iii) that interocular plasticity involving the visual cortex is significantly reduced in old animals support a cortical involvement in age-related visual decline in mice. They are consistent with the interpretation that in old age, the visual cortex loses its capacity to compensate for weaker inputs from the sensory periphery, apparent e.g., in a reduced electroretinogramme amplitude (Li et al., 2001; Gresh et al., 2003; Williams and Jacobs, 2007). In young adult mice mutant for the presynaptic scaffolding protein Bassoon, transmission at the photoreceptor ribbon synapse is severely impaired due to floating or absent ribbons (Dick et al., 2003) and visual acuity is significantly reduced compared to wildtype littermates (Goetze et al., 2010). Surprisingly, however, visual cortical activity maps recorded by intrinsic signal optical imaging were indistinguishable from wildtypes indicating that the central stages of visual processing, including the cortex, must have an extraordinarily high potential to process and compensate for altered inputs from the retina. It therefore appears that the reduced cortical activity observed in the present study in old mice, rather than being a consequence of deteriorated ocular input, develops independently of decreases in afferent activity and is rather due to mechanisms intrinsic to the cortex. Indeed, such changes have been documented both anatomically and physiologically: there is an approximately $20 \%$ loss of neurons in the visual cortex of aged rats (Yates et al., 2008) that may explain lower signal amplitudes in the visual cortex; in addition, a decline in cortical inhibition has been suggested as an explanation for age-related impairments in sensory processing. In the visual cortex of old cats, the number of GABAergic neurons is markedly reduced (Hua et al., 2008), and GABA agonists applied to visual cortical neurons of old monkeys restore orientation tuning, peak responses and signal-to-noise ratios of these neurons to young adult values (Leventhal et al., 2003). In the auditory cortex of rats, the RNA and protein levels of glutamic acid decarboxylase, the GABA synthesizing enzyme, decline with age (Ling et al., 2005). At first glance, our finding of a lower retinotopic map amplitude in old mice may seem to contradict the notion of a reduced cortical inhibition. Signal detection by optical imaging, however, relies on relative changes of activity. As the lack of inhibition may entail a lack of specificity 
and a decreased signal-to-noise ratio, optical imaging could reveal reduced activity.

\subsection{Increased interindividual variance during aging suggests environmental and life history influences}

The observation of a pronounced variability in interocular plasticity in old mice suggests that the decline in plasticity is not only due to organismic age-related processes, but also influenced by individual life-history events or environmental factors. This conclusion is also supported by the observation of pronounced variability of individual ocular dominance indices obtained by intrinsic signal optical imaging in old mice. Inflammatory processes have recently been implicated in age-related cognitive decline (Sparkman and Johnson, 2008; Witte et al., 2009) and might be subject to huge interindividual variability depending on housing environment, life style, diet and health status. Indeed, the observation that animals which were tested longitudinally in both the VWT and the optomotor system had visual acuities at 27 months of age that were in the uppermost range of animals tested just once - and never in the VWT - strongly suggests that physical exercise and/or environmental enrichment help to preserve visual functions. If decreasing cortical activity is an important factor for age-related visual decline, then interventions that stimulate the brain, like physical or mental exercise, should be beneficial for visual function. Only few studies to date have investigated this relationship in human subjects and indeed found a positive effect of physical activity on visual acuity (Vlahov, 1977), myopia in young students (Jacobsen et al., 2008) and visual attention in old adults (Roth et al., 2003). Thus, new therapeutic approaches for vision loss in ageing humans might be directed not at the eyes, but at the brain.

\section{ACKNOWLEDGMENTS}

The authors wish to thank Bianka Götze, Franziska Greifzu and Naira Yeritsyan for help in some of the experiments. Thanks are also due to Elke Woker and Anne-Kathrin Pilz for technical assistance and excellent animal care. Support of the BMBF is gratefully acknowledged.

\section{Disclosure statement}

The authors declare that they have not conflict of interest.

\section{References}

Birch, D.G. and Anderson, J.L. (1992). Standardized full-field electroretinography. Normal values and their variation with age. Arch Ophthalmol, 110, 1571-1576.

Cang, J., Kalatsky, V.A., Löwel, S. and Stryker, M.P. (2005a). Optical imaging of the intrinsic signal as a measure of cortical plasticity in the mouse. Vis Neurosci, 22, 685-691.

Cang, J., Kaneko, M., Yamada, J., Woods, G., Stryker, M.P. and Feldheim, D.A. (2005b). Ephrin-as guide the formation of functional maps in the visual cortex. Neuron, 48, 577-589.

David-Jürgens, M., Churs, L., Berkefeld, T., Zepka, R.F. and Dinse, H.R. (2008). Differential effects of aging on fore- and hindpaw maps of rat somatosensory cortex. PLoS One, 3, e3399.

Dick, O., tom Dieck, S., Altrock, W.D., Ammermüller, J., Weiler, R., Garner, C.C., Gundelfinger, E.D. and Brandstätter, J.H. (2003). The presynaptic active zone protein bassoon is essential for photoreceptor ribbon synapse formation in the retina. Neuron, 37, 775-786.

Douglas, R.M., Alam, N.M., Silver, B.D., McGill, T.J., Tschetter, W.W. and Prusky, G.T. (2005). Independent visual threshold measurements in the two eyes of freely moving rats and mice using a virtual-reality optokinetic system. Vis Neurosci, 22, 677 684.

Elliott, D.B., Yang, K.C. and Whitaker, D. (1995). Visual acuity changes throughout adulthood in normal, healthy eyes: Seeing beyond 6/6. Optom Vis Sci, 72, 186-191.

Ferrer-Blasco, T., Gonzalez-Meijome, J.M. and Montes-Mico, R. (2008). Age-related changes in the human visual system and prevalence of refractive conditions in patients attending an eye clinic. J Cataract Refract Surg, 34, 424-432.

Frisén, L., and Frisén, M. (1981). How good is normal visual acuity? A study of letter acuity thresholds as a function of age. Albrecht Von Graefes Arch Klin Exp Ophthalmol, 215, 9.

Godde, B., Berkefeld, T., David-Jürgens, M. and Dinse, H.R. (2002). Age-related changes in primary somatosensory cortex of rats: Evidence for parallel degenerative and plastic-adaptive processes. Neurosci Biobehav Rev, 26, 743-752.

Goetze, B., Schmidt, K.F., Lehmann, K., Altrock, W.D., Gundelfinger, E.D. and Löwel, S. (2010). Vision and visual cortical maps in mice with a photoreceptor synaptopathy: reduced but robust visual capabilities in the absence of synaptic ribbons. Neuroimage, 49, 1622-1631.

Gordon, J.A. and Stryker, M.P. (1996). Experience-dependent plasticity of binocular responses in the primary visual cortex of the mouse. J Neurosci, 16, 3274-3286.

Gresh, J., Goletz, P.W., Crouch, R.K. and Rohrer, B. (2003). Structure-function analysis of rods and cones in juvenile, adult, and aged C57bl/6 and Balb/c mice. Vis Neurosci, 20, 211-220. 
Hensch, T.K. (2005). Critical period plasticity in local cortical circuits. Nat Rev Neurosci, 6, 877-888.

Hua, T., Kao, C., Sun, Q., Li, X. and Zhou, Y. (2008). Decreased proportion of GABA neurons accompanies age-related degradation of neuronal function in cat striate cortex. Brain Res Bull, $75,119-125$.

Hua, T., Li, X., He, L., Zhou, Y., Wang, Y. and Leventhal, A.G. (2006). Functional degradation of visual cortical cells in old cats. Neurobiol Aging, 27, 155-162.

Jacobsen, N., Jensen, H. and Goldschmidt, E. (2008). Does the level of physical activity in university students influence development and progression of myopia?-a 2-year prospective cohort study. Invest Ophthalmol Vis Sci, 49, 13221327.

Kalatsky, V.A. and Stryker, M.P. (2003). New paradigm for optical imaging: temporally encoded maps of intrinsic signal. Neuron, $38,529-545$.

Lehmann, K. and Löwel, S. (2008). Age-dependent ocular dominance plasticity in adult mice. PLOS ONE, 3, e3120.

Leventhal, A.G., Wang, Y., Pu, M., Zhou, Y. and Ma, Y. (2003). GABA and its agonists improved visual cortical function in senescent monkeys. Science, 300, 812-815.

Li, C., Cheng, M., Yang, H., Peachey, N.S. and Naash, M.I. (2001). Age-related changes in the mouse outer retina. Optom Vis Sci, $78,425-430$

Liguz-Lecznar, M., Siucinska, E., Zakrzewska, R. and Kossut, M. (2011) Impairment of experience-dependent cortical plasticity in aged mice. Neurobiol Aging, 32, 1896-1905.

Ling, L.L., Hughes, L.F. and Caspary, D.M. (2005). Age-related loss of the GABA synthetic enzyme glutamic acid decarboxylase in rat primary auditory cortex. Neuroscience, 132, 1103-1113.

Mendelson, J.R. and Ricketts, C. (2001). Age-related temporal processing speed deterioration in auditory cortex. Hear Res, 158, 84-94.

Owsley, C., Sekuler, R. and Siemsen, D. (1983). Contrast sensitivity throughout adulthood. Vision Res, 23, 689-699.

Porciatti, V., Burr, D.C., Morrone, M.C. and Fiorentini, A. (1992). The effects of aging on the pattern electroretinogram and visual evoked potential in humans. Vision Res, 32, 1199-1209.

Prusky, G.T., Alam, N.M., Beekman, S. and Douglas, R.M. (2004). Rapid quantification of adult and developing mouse spatial vision using a virtual optomotor system. Invest Ophthalmol Vis Sci, 45, 4611-4616.

Prusky, G.T., Alam, N.M. and Douglas, R.M. (2006). Enhancement of vision by monocular deprivation in adult mice. J Neurosci, $26,11554-11561$.

Prusky, G.T., West, P.W. and Douglas, R.M. (2000). Behavioral assessment of visual acuity in mice and rats. Vision Res, 40, 2201-2209.
Roth, D.L., Goode, K.T., Clay, O.J. and Ball, K.K. (2003). Association of physical activity and visual attention in older adults. $J$ Aging Health, 15, 534-547.

Schmolesky, M.T., Wang, Y., Pu, M. and Leventhal, A.G. (2000). Degradation of stimulus selectivity of visual cortical cells in senescent rhesus monkeys. Nat Neurosci, 3, 384-390.

Sparkman, N.L. and Johnson, R.W. (2008). Neuroinflammation associated with aging sensitizes the brain to the effects of infection or stress. Neuroimmunomodulation, 15, 323-330.

Spear, P.D. (1993). Neural bases of visual deficits during aging. Vision Res, 33, 2589-2609.

Tomoda, H., Celesia, G.G., Brigell, M.G. and Toleikis, S. (1991) The effects of age on steady-state pattern electroretinograms and visual evoked potentials. Doc Ophthalmol, 77, 201-211.

Trachimowicz, R.A., Fisher, L.J. and Hinds, J.W. (1981). Preservation of retinal structure in aged pigmented mice. Neurobiol Aging, 2, 133-141.

van Alphen, B., Winkelman, B.H. and Frens, M.A. (2009). Age- and sex-related differences in contrast sensitivity in C57BL/6 mice. Invest Ophthalmol Vis Sci, 50, 2451-2458.

Vlahov, E. (1977). Effect of the Harvard Step Test on visual acuity. Percept Mot Skills, 45, 369-370.

Wang, H., Xie, X., Li, X., Chen, B. and Zhou, Y. (2006). Functional degradation of visual cortical cells in aged rats. Brain Res, 1122 , 93-98.

Weleber, R.G. (1981). The effect of age on human cone and rod ganzfeld electroretinograms. Invest Ophthalmol Vis Sci, 20, 392-399.

Williams, G.A. and Jacobs, G.H. (2007). Cone-based vision in the aging mouse. Vision Res, 47, 2037-2046.

Witte, A.V., Fobker, M., Gellner, R., Knecht, S. and Floel, A. (2009). Caloric restriction improves memory in elderly humans. Proc Natl Acad Sci U S A, 106, 1255-1260.

Wolf, N.S., Li, Y., Pendergrass, W., Schmeider, C. and Turturro, A. (2000). Normal mouse and rat strains as models for age-related cataract and the effect of caloric restriction on its development. Exp Eye Res, 70, 683-692.

Wong, A.A. and Brown, R.E. (2007). Age-related changes in visual acuity, learning and memory in C57BL/6J and DBA/2J mice. Neurobiol Aging, 28, 1577-1593.

Yates, M.A., Markham, J.A., Anderson, S.E., Morris, J.R. and Juraska, J.M. (2008). Regional variability in age-related loss of neurons from the primary visual cortex and medial prefrontal cortex of male and female rats. Brain Res, 1218, 1-12. 